\title{
The effect of cell boundary on 2D foam
}

\author{
Atef AbdelKader ${ }^{1 *}$ \\ ${ }^{1}$ Department of Mathematics and Science, Ajman University, UAE.
}

\begin{abstract}
We have studied the effect of cell boundary on 2D foam, with particular attention to perfect arrays of identical bubbles, and those containing only a single defect with time. We have also examined the effect of the wetness of the foam, observing the stability of two-dimensional foam comprising bubble rafts constrained to a fixed area of liquid surface. Perfectly six-fold coordinated foam appear to be unstable against loss of cohesion, but the lifetime to breakage of the perfect foam increases systematically with changing the cell boundaries. Foams containing a single defect are stable against such breakage due to the elastic stress fields around it.
\end{abstract}

\section{Introduction}

Relatively ordered foam exhibits an initial transient in its evolution [1], [2], [3] which has been interpreted in terms of the growth of disorder from individual topological defects. The past decades has witnessed increasing interests in the study of such growing disorder. Considerable attention was addressed to the scaling state by many authors and it has been studied experimentally and numerically [4], [5], and [6]. Levitan investigated the evolution of an isolated defect in an otherwise ideal hexagonal froth [7] concentrating on the cluster of disordered bubbles around the initial defect (the set of bubbles having at least one nonhexagonal neighbour). As cluster of transformed bubbles grow; the time dependence of the number of bubbles in this cluster in investigated and the distribution of the topological classes in the evolving part of the system is calculated. The results of the latter study challenged the common wisdom that the scaling state dynamics does not depend on the initial condition, suggesting as they did, that the long-time topological distribution function, while of stable form, differed from that for generic initial conditions (random 2D froth). Not in standing with, considerable controversy has been initiated [8], [9] and subsequent computer simulations have been carried out suggesting that more conventional ideas are more likely correct [10], [11]
,[12], [13]. In the present work, we report an experimental study of such perfectly triangular lattices, as well as ones containing a dislocation defect.

\section{Experimental method}

The simulations discussed above concern dry 2D froth. While it is possible to make 2D froth which is nearly dry [14], it appears essentially impossible to create perfectly ordered froth. Two-dimensional arrays of bubbles on the surface of soap solutions have been exploited as useful models of various aspects of condensed matter physics, following the pioneering work of Bragg [15], [16]. We have adapted these bubble rafts to permit formation of perfectly ordered 2D foam. Fortes et al. [17], have demonstrated that bubble rafts trapped between the surface of a soap solution and a glass cover plate endure essentially indefinitely, temporal evolution being restricted to that due to coarsening. Restricting such $2 \mathrm{D}$ foam to a hexagonal shape helps enforce the desired symmetry. The glass cover is supported just $2 \mathrm{~mm}$ above the soap solution on a metal plate which contains a hexagonal hole (typically $6 \mathrm{~cm}$ on a side). The plate extends into the solution and 2D foam is formed by bubbling N2 into the solution below this hexagonal cell via a long hypodermic needle. By systematically sweeping the tip of the needle to and fro as the lines of bubbles form it is possible, with practice, to create perfectly 6 -fold 
coordinated lattices comprising several thousand bubbles about $2 \mathrm{~mm}$ in diameter. A dislocation is introduced by interrupting the process when the lattice is part made, and then completing the lattice as shown in Fig. 1. This process has been repeated several times using hexagonal frame with elastic boundaries, less wet bubbles, and also by changing the depth between the glass cover and the soap solution. The 2D foams produced as above are of course wet, but we may hope that their behavior may reflect at least some of the generic aspects of the evolution of $2 \mathrm{D}$ froth as revealed in the simulations [10], [11].

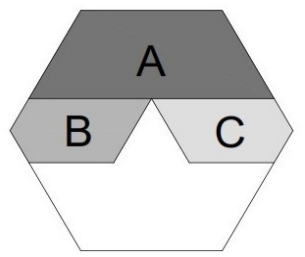

Fig. 1. A sketch outlining the procedure for creating a defect. A crystalline lattice is first made in region $\mathrm{A}$, then regions $\mathrm{B}$ and $\mathrm{C}$ are filled with bubbles in register with those in $\mathrm{A}$, leaving a gap. This fills in as the remaining space is filled with bubbles, creating a dislocation.

\section{Results and discussion}

Most of the data shown are bubbles in the order $2 \mathrm{~mm}$ in diameter in a cell $6 \mathrm{~cm}$ on a side. We mainly consider the effects of boundaries of the system as well as the effect of the wetness of the foam.

The ideal 6-fold coordinated 2D foam shows no great change for the stability of the foam a period of order 25 minutes and then breaks, creating an area that lacking any bubbles as shown in Fig. 2. (a)

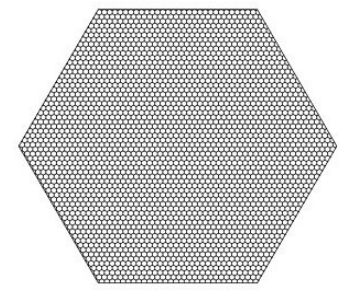

(b)

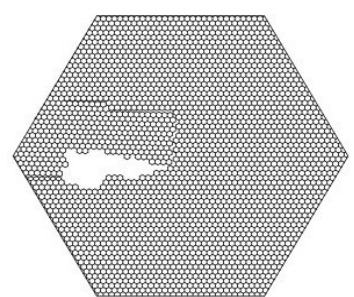

Fig. 2. Pictures of ideal $2 D$ foam. (a) The $2 D$ foam as formed $(t=0)$ and $(b) t=25$ minutes after creation.

During the rupture, the number of bubbles is conserved. The system is clearly under tension and the breakage consists of a crack appear between a few bubbles which then spread rapidly to form a large and ultimately stable hole in the foam. When the boundary of the cell is replaced by more-or-less elastic one, the lifetime of the cell has increased up to 100 minutes as shown in Fig. 3. When the dry bubbles are used, a relatively long lifetime is indicated. Experiments with hexagonal cells that are $3 \mathrm{~cm}$ and $10 \mathrm{~cm}$ on a side indicate that the system size has no effect upon the phenomena.

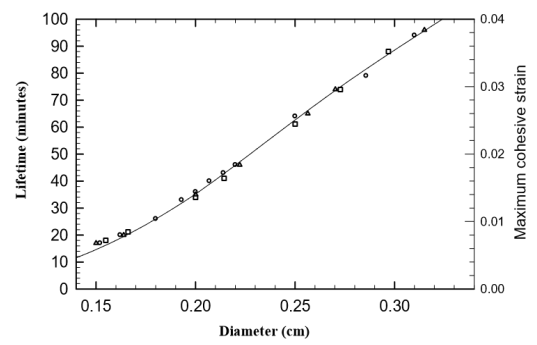

Fig. 3. Variation of lifetime of perfect foam with bubble diameter in hexagonal cells of different sizes using elastic boundary. The line represents the variation of the cohesive strain with the bubble diameter.

However, the lifetime to breakage of the perfect foam increases systematically with bubble size comparing with that obtained by using a hard boundary and wet foam as shown in Fig. 4.

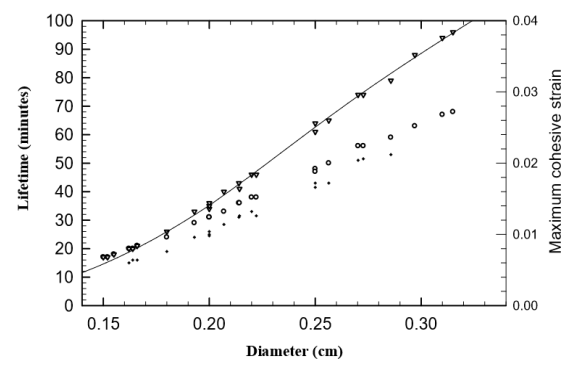

Fig. 4. Variation of lifetime of perfect foam with bubble diameter in hexagonal cells of different sizes for elastic and hard boundary. The line represent the variation of the cohesive strain with the bubble diameter.

Optional introduction of a dislocation into 2D foam as shown in Fig. 5, (either located close to a boundary or more centrally), drastically alters the system's evolution. These differences in behaviour were completely unexpected. At this stage of manuscript preparation, authors are not aware of any other experimental studies of the long-time behaviour of ideal 2D foam including defects. It is suggested that stabilization arises from the deformation of the bubbles in response to the stress field surrounding the defect. The quadratic dependence of the surface energy of the bubbles on the deformation leads to a contribution to the system energy which increases as the system incorporating a defect attempts to approach equilibrium packing. Turning to the $2 \mathrm{D}$ foam containing a single defect, the results appear to be independent of system size, at least over the entire range indicated as above. A 
typical example of the evolution of a single defect in an otherwise regular hexagonal lattice is shown in Fig. 5.

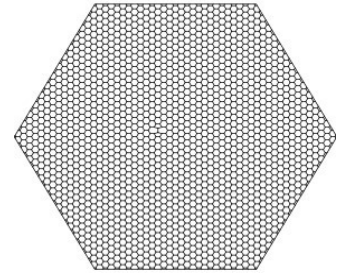

Fig. 5. 2D foam containing a single dislocation as made where no breakage has occurred.

The initial cluster grows as the disorder increases around the initial defect due to coarsening. Essentially no observable changes occur in the system for 12-15 hours, about which time the first increase in disorder around the defect appears.

We study the evolution of a 'cluster', defined as all bubbles having at least one neighbour, which is not 6fold coordinated [7], [10]. We state here that in the first stage, the whole bubbles in the cluster are having six-coordinates. The stability of the foam has been followed by investigating the evolution of certain statistics of the cluster. These include the distribution of the so-called topological classes (bubble coordination numbers) and its second moment. The tail of the topological distribution function, $\mathrm{P}(\mathrm{n})$, was extended towards larger values of $\mathrm{n}$ as time progressed, while the peak of the distribution is kept at $n=6$ (Fig. 6 ). Turning into quantitative measures, the second moment of the topological class distribution of the cluster $(\mu 2)$ grew monotonically with time (Fig. 7), reaching rather larger values when less wet bubbles is used as well as a hard boundaries. Here we compare current data with the data obtained when we used wet bubbles and an elastic boundary. We found that $(\mu 2)$ dropped to lower value when we used soft boundaries. On the other hand, higher values of $(\mu 2)$ are reported when we use less wet bubbles as expected. Paying attention to the perfect foam, an answer to the ordinal question of why the diameter and the wetness of the bubbles affected the lifetime should be provided. In that respect, we consider our ideal foam as perfect in the sense that all bubbles are 6-fold coordinated even though it should be noted that these bubbles must also have small variations in both size and separation. The latter lead to small readjustments of the positions of the bubbles in which the system locally seeks to reach equilibrium at $\mathrm{r}=\mathrm{r} 0$ (Fig. 8). The bubbles thus become closer in some places and more separated in others. In that sense, the global energy of the system must reduce. As this process continues, the local stresses accumulate at various points, of which few are becoming weak. As the local strain reaches a critical level, the cracks appear in this area just one or two minutes before the breakage of the foam. This critical strain is just the strain at which the stress (force/bubble) is maximum

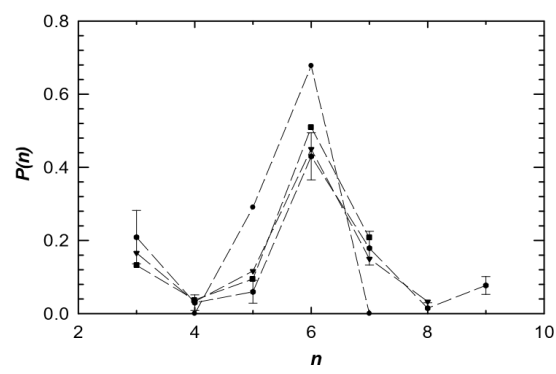

Fig. 6. Topological class distribution $\mathrm{P}(\mathrm{n})$ for evolving foam containing an isolated defect in several experiments.

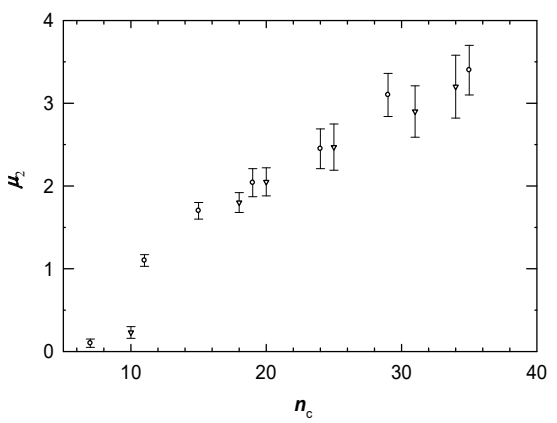

Fig. 7. Variation of $\mu_{2}$, the second moment of the cluster with $\mathrm{nc}$ as different foams (different symbols) containing a dislocation evolve.

If the internal tension exceeds this value, the lattice of bubbles will rupture. This corresponds to the cohesive strain of the system (defined as the ratio of the separation between $\mathrm{r} 0$ and that $\mathrm{r}$ corresponding to maximum resistive force (i.e. maximum $\delta \mathrm{V}(\mathrm{r}) / \delta \mathrm{r}$ ) and the bubble diameter), which increases monotonically with bubble size [19]. The cohesive strain between two bubbles was defined as the ratio of the critical separation distance, between the position of initial equilibrium and the position of maximum resistive force on the pair force-displacement law, to the diameter of the free bubble. Here we consider the dependence of the lifetime of the ideal foam upon the bubble size. We mentioned that the lifetime of the ideal foam increases systematically with the bubble size, in qualitative agreement with the variation of the cohesive strain. When the bubbles become larger, the breakage of the foam requires a large strain. A long time is needed for the local strain to accumulate to such a large value, so the lifetime increases. It is difficult to compute the cohesive strain in the present case quiet precisely. In addition, to our knowledge, the separation between the bubbles in the initial foam remain unjustified however it should rather exceeds ro. By far, the potential which is computed by Nicolson et. al [18] and Lomer et.al [20] is for a bubble raft with a free 
surface, where the top of each bubble being a spherical cap, whereas in our case the bubbles are pushed up against the flat cover glass, which the soap films must meet at $90^{\circ}$. The boundary conditions are thus not those used by previous workers. However, for the present approximate calculation we use Nicolson's potential. Lomer's more correct form [20] differs only in the repulsive contribution, which is not very significant at $r$ rather above $\mathrm{r} 0$, and is less simple to use. For a range of bubble sizes, we computed $\mathrm{r} 0$ and that $\mathrm{r}$ corresponding to maximum inter-bubble force, to calculate the cohesive strain. The general trend of the plot of the cohesive strain against the diameter of the bubbles shows very good general agreement with the variation of our lifetime data (see Fig. 3, 4). The displacement between the theoretical line and some of the experiment data may be due to the differences of our system from that treated by Nicolson discussed above. The lifetime is defined as the time required to accumulate strain to the maximum cohesive strain. In our result, the latter is not found to depend on the thickness of the air gap between soap solution and the cover glass. However, the violence of the foam breakage is observed to increase when the gap is reduced. Moreover, we deduce that the force on the bubbles depends on the air-gap, while the maximum cohesive strain does not. Statedother-wise, the meniscus must meet the cover plate at $90^{\circ}$ and become horizontal at large distances. The interbubble potential depends on the height of the meniscus. Thus, the force proportional to $\mathrm{dV}(\mathrm{r}) / \mathrm{dr}$, must increase accounting for the more curved meniscus for the thinner air gap.

\section{References}

1. Stavans and J.A. Glazier et al., Phys. Rev. Lett. 62. 1318 (1989).

2. J.A. Glazier, S.P. Gross, and J. Stavans, Phys. Rev. A 36, 306 (1987).

3. Stavans and J.A. Glazier et al., Phys. Rev. Lett. 62. 1318 (1989).

4. H. Flyvbjerg and C. Jeppesen, Phys. Scr. T38, 49 (1991).

5. V.E. Fradkov, L.S. Shvidlerman, and D.G. Udler, Philos. Mag. Lett. 55, 289 (1987).

6. B. Levitan, M.Sc. thesis (1992).

7. B. Levitan, Phys. Rev. Lett. Volume 72, No. 26, 4057 (1994).

8. Weaire, D., Phys. Rev. Lett. 74: 3710 (1994).

9. Sire, C. Phys. Rev. Lett. 74: (1995) 3708.
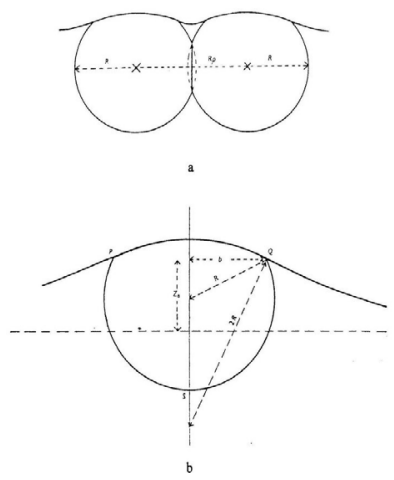

Fig. 8. (a) Two bubbles in contact. (b) Single bubble floating on liquid $\mathrm{PQ}$ is the ring of contact of radius $b$. The free surface is indicated by the broken line. (From [18]).

\section{CONCLUSIONS}

We show that arrays of bubbles constrained within a rigid cell are metastable with respect to breakage (loss of cohesion) if they are initially ideally six-fold coordinated. However, when the rigid cell replaced by more elastic one and when the foam is less wet, the lifetime increases relatively. The significant increasing in the lifetime is related to the elastic stress fields in the cell. Such foams are stabilized by the introduction of a single point defect, the temporal evolution that associated with disproportionate of gas between small and large bubbles. The evolution of a single defect in an isotropic hexagonal lattice has been investigated experimentally for comparison with previous study. In general, our results qualitatively support results from previous work.

10. Jiang, Y., Mombach, M., \& Glazier, J. A., Phys. Rev. E 52: (1995) 3333-3338.

11. Ruskin, H. J., Feng, Y., J. Phys. Condens. Matter 7: (1995) L553-562.

12. Levitan, L., Domany, E., Phys. Rev. E 54: (1996) 27662771.

13. Chae, J. J., Tabor, M., Phys. Rev. E 55: (1997) 598610. T38, 49 (1991).

14. Stavans, J., Phys. Rev. A 42: (1990) 5049-5053.

15. Bragg, W. L., Proc. Phys. Soc., 52: (1940) 54-56.

16. Bragg, W. L. J. Sci. Instrum., 19: (1942) 148-150.

17. Fatima Vaz, M., Fortes, M. A. J. Phys.: Condense Matter 9: (1997) 8921-8930.

18. Nicolson, Mathematical proceedings of the Cambridge Philosophical Society, Math. phys. Sci., 45: (1949) 288-293.

19. Shi, L. T., Argon, A. S., Philosophical Magazine A 46: (1982) 275-286.

20. Lomer, W., Proc. Royal Soc. London Series A, 196: (1949) 182-194. 